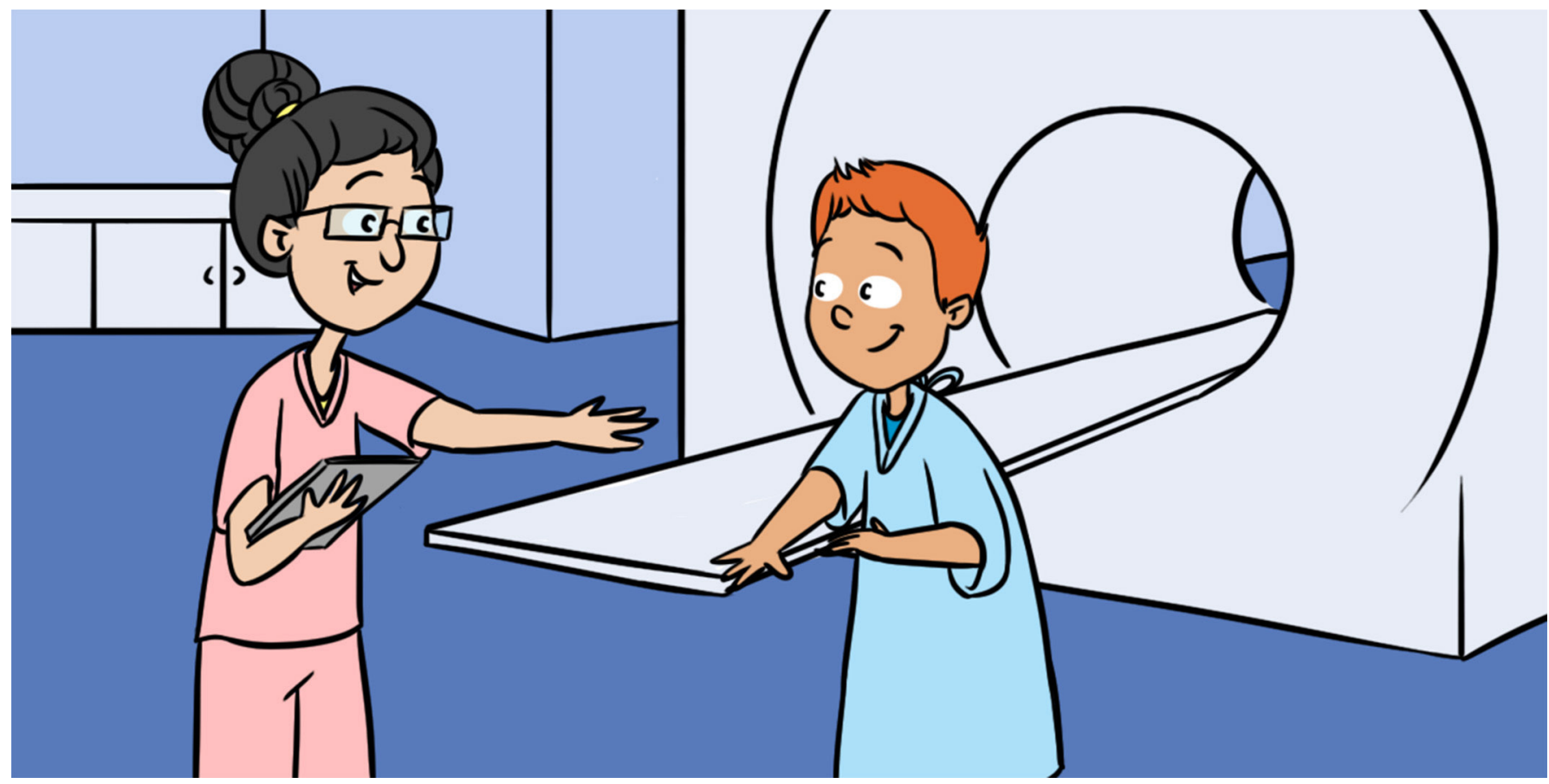

\title{
THE PHYSICS OF MRI AND HOW WE USE IT TO REVEAL THE MYSTERIES OF THE MIND
}

\section{Kathryn Mary Broadhouse*}

Sunshine Coast Mind and Neuroscience Thompson Institute, University of the Sunshine Coast, Sunshine Coast, QLD, Australia

\section{YOUNG REVIEWERS:}

\begin{tabular}{|l|}
\hline SAS \\
MATTHEW \\
FLINDERS \\
Anglican colege \\
\hline
\end{tabular}

MATTHEW FLINDERS ANGLICAN COLLEGE AGES: $14-15$
Our ability to interpret and process information and regulate emotion is determined by the structure and chemical makeup of our brains. A change to the structure or biochemical makeup of our brains can affect our mental health, the way we cope with the normal stresses of life, our productivity, and our overall well-being. Scientists interested in studying the brain and mental health disorders (called neuroscientists) seek to understand how the architecture and biochemical makeup of the brain differs in health and disease. To do this, we use a technique called magnetic resonance imaging (MRI) to image inside the living brain, to examine its function and structure. This article explains the physics of MRI and how we use it to generate images of our brains in order to investigate how the mind works.

\section{THE BRAIN: HOME OF THE MIND}

As the center of the nervous system, the brain makes sense of the information from our surroundings. The brain interprets information 
and both releases chemicals and sends electrical signals (messages) to cause the body to respond. However, our brains not only regulate our bodies and our movements but are also the home of our minds. The brain is the physical place where the mind resides.

Our minds constantly control the flow of information-our memories, thoughts, emotion, and imagination. This flow of information (Figure 1A) can be thought of as a social network where, instead of messages being sent between friends on social media, information is being sent to different parts of the brain along neurons. The mind's network relies on the underlying structure and chemical makeup of the brain to function successfully. In fact, a successful or effective mind network allows us to be cognitively (our ability to carry out mental activities), emotionally, and socially healthy.

\section{MENTAL HEALTH}

The mind network, the way it sends information and its underlying structure determine our mental health. In mental health disorders, such as depression and anxiety, we often see a disruption to the mind network (Figure 1B). This change to the underlying structure and chemical makeup of our brains can affect the way we can cope with the normal stresses of life, our productivity, and our overall wellbeing. Therefore, neuroscientists who want to understand mental health disorders and how we can all stay mentally healthy seek to examine the mind network. If we can understand how the brain's structure and chemical makeup lead to healthy function, then we can start to understand brain disorders. However, to be able to study the mind and its network, we need to be able to look inside people's brains. To do this we use MRI.

A) Flow of information our emotions, and our thoughts can be thought of as a social network where, instead of messages being sent between friends on social media, information is being sent to different parts of the brain along neurons. (B) In mental health disorders, such as depression and anxiety, we often see a disruption to the mind network. This disruption can affect the way the mind network sends, receives, or interprets messages.
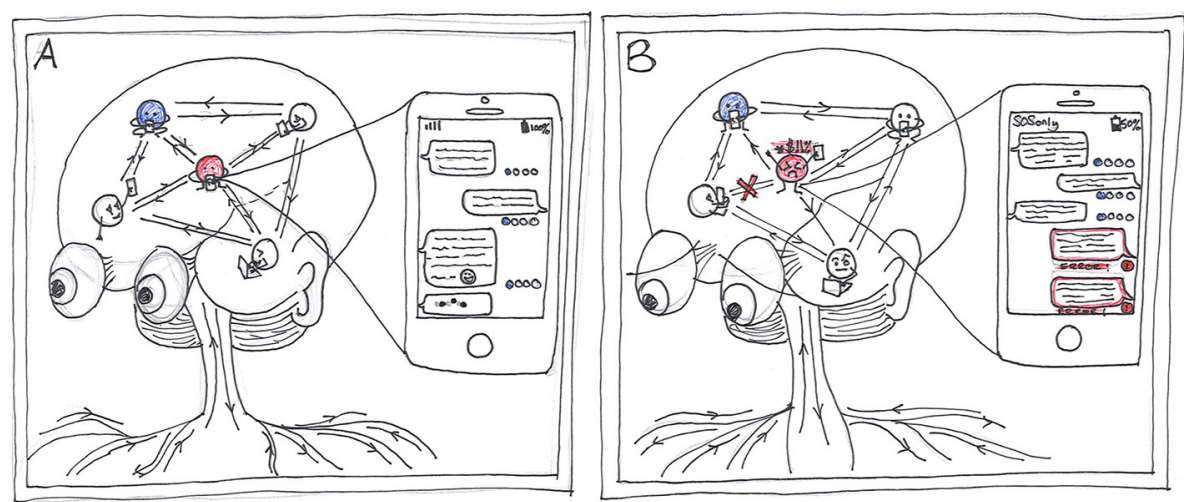

Figure 1 


\section{MAGNETIC RESONANCE IMAGING: MRI}

Magnetic resonance imaging (MRI) allows us to see inside the human body with amazing detail, by using magnets and radio waves. The first MRI scanner used to image the human body was built in New York in 1977. Since then, the technology has come a long way and MRI is now used frequently by doctors to look inside the human body. This is because MRI does not involve radiation (like an X-ray or CT scan) and there are more and more MRI scanners being installed around the world.

\section{THE "MAGNET" IN MAGNETIC RESONANCE IMAGING}

The MRI scanner is essentially a giant magnet. The strength of the magnet is measured in a unit called Tesla (T). Most MRI scanners used in hospitals and medical research clinics are 1.5 or $3 \mathrm{~T}$. Putting that in to

Figure 2

Hydrogen protons and how they behave in a magnetic field.

(A) Water is made up of two hydrogen atoms and one oxygen atom. The hydrogen nucleus (shown in red as $\mathrm{P}+$ ) contains one positive charge-a proton spinning around on its axis, which acts like a tiny magnet. (B) In the MRI scanner, the protons align with the BO magnetic field, some "up" (red), and slightly less "down" (white). The total magnet field generated from all the hydrogen protons almost cancel each other out, to leave only the magnetic field from the small proportion of extra "up" protons, and it is this small magnetic field that we can measure using MRI. (C) When an RF wave/pulse with the same frequency as the proton's precessional frequency is turned on, the "up" protons flip away from the BO field, as they absorb the RF energy. perspective, the earth's magnetic field is around $0.00006 \mathrm{~T}$. A 3 T MRI scanner is around 60,000 times stronger than the earth's magnetic field!

MRI uses magnetic fields and radio waves to measures how much water is in different tissues of the body, maps the location of the water and then uses this information to generate a detailed image. The images are so detailed because our bodies are made up of around $65 \%$ water, so we have lots of signal to measure. The water molecule $\left(\mathrm{H}_{2} \mathrm{O}\right)$ is made up of two hydrogen atoms and one oxygen atom. The hydrogen $(\mathrm{H})$ atoms are the part that makes water interesting for MRI, and what we use to measure the signal from the body when we do an MRI scan.

If we look at hydrogen in more detail, we see it has a central nucleus containing a single positive charge, called a proton (Figure 2A). Like the Earth spinning on its axis with a north and south magnetic pole,

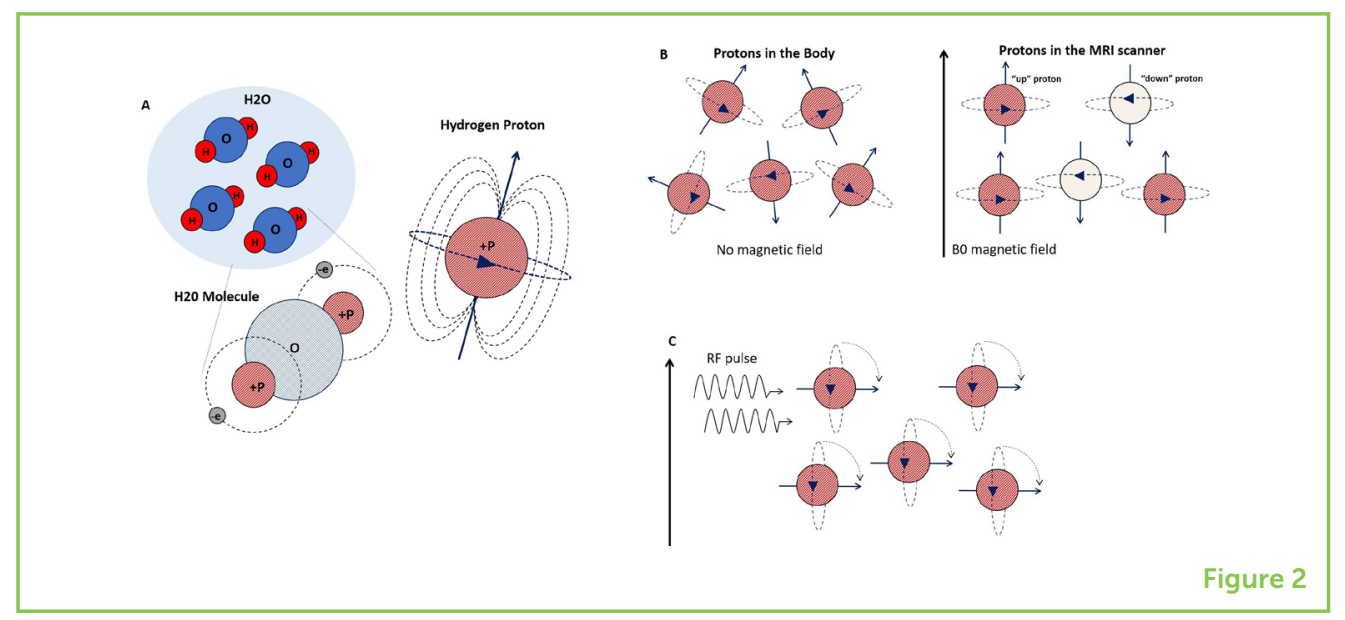


BO

The MRI scanner's main magnetic field.

PRECESSIONAL

FREQUENCY

The rate at which protons spin in a magnetic field.

RF

Radio frequency pulse used to tip on resonance protons away from the BO field.

ON RESONANCE

Have the same frequency. each spinning hydrogen proton is like a tiny magnet that spins around on its own axis. This spinning motion is known as precession. At any moment in time, all the billions of hydrogen protons in our bodies are all in random positions and spinning on their axes.

However, this randomness changes when we place a human body into a very strong magnetic field, like an MRI scanner. Just like a compass needle aligns to the Earth's magnetic field, when these randomly spinning hydrogen protons are placed in an MRI scanner, their axes realign them with the scanner's stronger magnetic field. We call the scanners magnetic field the BO field. Just like a compass needle in the earth's field, the compass itself does not physically move, but rather the needle spins to align itself. Similarly, the hydrogen protons do not physically move in your body when you enter an MRI scanner, their axes just align along the direction of the BO field. Some will align "up" (parallel) and some will align "down" (anti-parallel), while still spinning around on their own axes (Figure 2B). Due to the wonderful laws of quantum physics, which we will not go into here, there are always just slightly more "up" protons than "down." If you now think about the total magnet field generated from all our hydrogen protons, these tiny magnets almost cancel each other out, to leave only the magnetic field from the small proportion of extra "up" protons, and it is this small magnetic field that we can measure using MRI.

The BO field not only affects the hydrogen proton's alignment, but also affects how fast these protons spin (called precessional frequency). The precessional frequency depends on the strength of the magnetic field. The stronger the magnetic field, the faster they spin. These two ideas of axis realignment and precessional frequency are important when we use MRI to measure the signal from these hydrogen molecules.

\section{HOW DO WE DETECT THE MAGNETIC FIELD?}

So how do we distinguish the tiny magnetic field caused by the extra "up" hydrogen protons in our body from the massive B0 field of the scanner? We use something called a radio frequency (RF) pulse, to disturb or flip all the protons, at the same time, out of alignment from the scanners magnetic field (Figure 2C). The frequency of the RF pulse must be the same as the frequency of the spinning hydrogen protons, so they can exchange energy, so that they are on resonance with each other. Resonance enables the protons to absorb enough energy from the RF pulse to rotate their axes away from the BO field, so that the MRI scanner can measure it. If we think again about our compass in the Earth's magnetic field pointing toward the north pole, we can make 
Figure 3

Focusing our imaging.

(A) The B1 field is applied, which increases across the body, from foot to head. Hydrogen protons in the head wil then be spinning faster than those in the feet.

(B) Different tissues, such as white matter, gray matter and cerebral spinal fluid (CSF) in the body give off different amounts of energy. To measure the energy emitted by the protons in the brain when the RF pulse is turned off, we place a coil around the head.

(C) This technique can provide many different images of the brain, giving us information about (top right): how the brain is structurally connected via white matter-the information highways of the brain (Middle right): the volume of gray matter regions of the brain, where information is processed. (Bottom right): how the brain is functionally connected-how different regions of the brain communicate and work together.

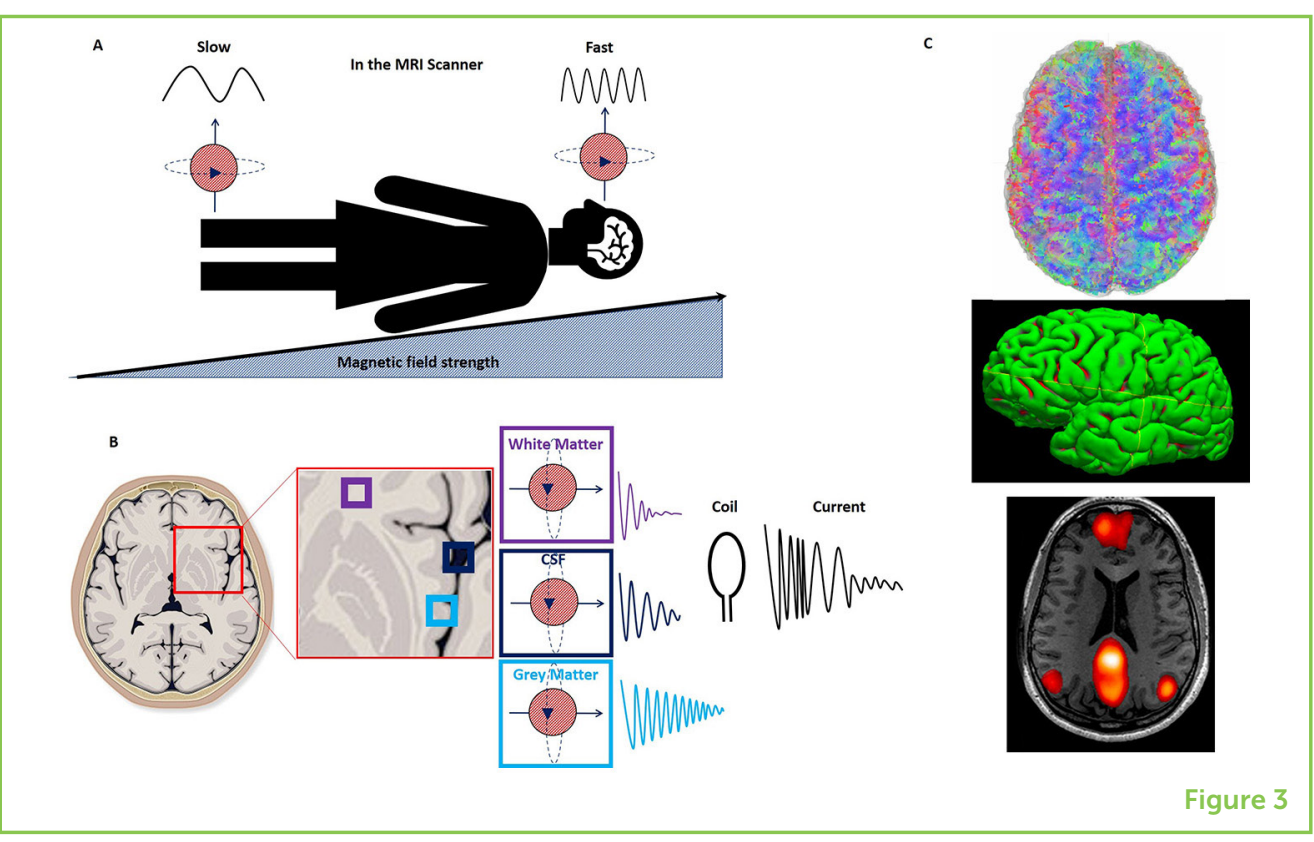

the needle rotate to point east if we place a small bar magnet next to the compass. This is similar to the way the protons behave when we turn on the RF pulse.

If the whole body is full of hydrogen "up" protons all spinning at the same precessing frequency in the $\mathrm{B0}$, how do we target just the ones in the brain to investigate mental health? We use the fact that the precessional frequency of the protons is dependant on the magnetic field strength. We apply a second magnetic field, B1 that varies across the body. In the example shown in Figure 3A, hydrogen protons in the head will then be spinning faster than those in the chest, stomach and feet. Then, we tune the RF pulse to the precessing frequency of the hydrogen protons in the head. The RF pulse will then only be resonant with the protons in the brain. Therefore, only the protons in the brain will absorb energy from the RF pulse and be flipped away from the BO field. We can obviously tune our RF pulse to be resonant with protons in other parts of the body, like the feet, if we were interested in imaging the feet!

\section{HOW DO WE GET AN IMAGE FROM THESE SPINNING PROTONS?}

So how do we get an image from these spinning, flipped hydrogen protons in the brain? When the RF pulse is turned off, the protons flip back and realign along the main magnetic field, BO. If we think of our compass again, when we move our small bar magnet away, the needle will rotate from east to north and align with the Earth's magnetic field once more. As the protons flip back and realign with B0, they give off energy. 
FOURIER

\section{TRANSFORM}

A mathematical calculation that is used to change the electrical current in a coil into an image.
Different tissues in the body give off different amounts of energy. To measure this emitted energy, we require some special equipment (called a coil) that is placed around the body part we are imaging (Figure 3B). The coil acts as an antenna and detects the released energy as an electrical current. The electrical current is transformed, via a computer, using a mathematical calculation called a Fourier transformation. Because protons in the different kinds of tissues in the brain, such as gray matter, white matter and blood, all give off different amounts of energy, the result of the transformed energy is a highly detailed image of the tissue inside the brain.

\section{CONCLUSION}

We have now described how we use MRI to generate and measure signal from water molecules in the body. But as well as providing images of the inside of our bodies, MRI can also be used to answer many different questions about the makeup of the brain and how it functions. From the MRI images, we can investigate not only the structure and chemical makeup of the brain, but also how our mind's network is connected and how different regions of the brain communicate with each other (Figure 3C). In this manner neuroscientists can investigate how the mind's network is different in health and mental health disorders. By understanding how mental health disease changes the brain we can begin to develop treatment for these disorders.

SUBMITTED: 27 September 2018; ACCEPTED: 04 February 2019; PUBLISHED ONLINE: 01 March 2019.

EDITED BY: Daniel F. Hermens, Sunshine Coast Mind and Neuroscience Thompson Institute, University of the Sunshine Coast, Australia

CITATION: Broadhouse KM (2019) The Physics of MRI and How We Use It to Reveal the Mysteries of the Mind. Front. Young Minds 7:23. doi: 10.3389/frym.2019.00023

CONFLICT OF INTEREST STATEMENT: The author declares that the research was conducted in the absence of any commercial or financial relationships that could be construed as a potential conflict of interest.

COPYRIGHT @ 2019 Broadhouse. This is an open-access article distributed under the terms of the Creative Commons Attribution License (CC BY). The use, distribution or reproduction in other forums is permitted, provided the original author(s) and the copyright owner(s) are credited and that the original publication in this journal is cited, in accordance with accepted academic practice. No use, distribution or reproduction is permitted which does not comply with these terms. 


\section{YOUNG REVIEWERS}

\section{MATTHEW FLINDERS ANGLICAN COLLEGE, AGES: 14-15}

Matthew Flinders Anglican College young reviewers are keen budding scientists who enjoy taking on challenges. They are a collaborative bunch of young minds, with shared interests in science, music, and sport. This group of young reviewers thoroughly enjoy being part of Frontiers for Young Minds and are excited to be involved in how science is being communicated and shaped.

\section{AUTHOR}

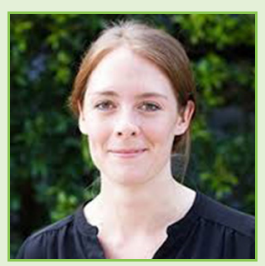

\section{KATHRYN MARY BROADHOUSE}

I decided I wanted to study Science and more specifically Physics at high school as I loved to learn how things and the universe work. When deciding my career, it was really important for me to be doing what I love, but also feel my work was meaningful and helping society. I therefore decided to specialize in medical imaging as this field combines a lot of the fundamental principles of physics to investigate and understand disorders and disease. *kathryn.broadhouse@usc.edu.au 